\title{
Implementation of Level-1 trigger algorithms on the upgraded CMS Global Trigger System
}

\author{
T. Matsushita; B. Arnold ', H. Bergauer, N. Rad \\ Institute of High Energy Physics, Austrian Academy of Sciences \\ Nikolsdorfer Gasse 18, 1050 Wien, Austria \\ E-mail: Takashi.Matsushita@oeaw.ac.at
}

\begin{abstract}
The Global Trigger is the final step of the CMS Level-1 Trigger and implements a trigger menu, a set of selection requirements applied to the final list of objects from calorimeter and muon triggers. The conditions for trigger object selection including topological requirements on multiobject triggers, invariant-mass calculations and other complex operations are combined by simple combinatorial logic to form the algorithms. To improve the performance of the Level-1 trigger system at high luminosity with large numbers of pile-up events expected at LHC Run 2, the electronics for the calorimeter trigger, muon trigger and Global Trigger will be replaced. The upgraded system will be flexible for implementing further rate reduction and efficiency improvements as algorithms improve. The flexibility will be accomplished by using high bandwidth optical links for most of the data communication between trigger cards, and by using modern, large FPGAs and large memory resources for the trigger logic. In order to handle the increased complexity of the trigger menu to be implemented on the upgraded Global Trigger as well as to make the menu implementation more flexible, a set of rules has been defined to express Level-1 trigger algorithms. The system to realise the trigger menu on FPGA from Level-1 trigger algorithms expression based on the rules has been developed. The design and implementation of the system for preparing a menu for the upgraded CMS Global Trigger system will be presented.
\end{abstract}

The European Physical Society Conference on High Energy Physics

22-29 July 2015

Vienna, Austria

*Corresponding author

† Speaker. 


\section{Introduction}

The CMS experiment has a two-level online event selection system. The hardware trigger (Level-1 trigger) is based on coarse information from the calorimeters and the muon detectors while the software trigger (High-Level Trigger) makes decisions with information from all subdetectors. Figure 1 shows block diagram of the CMS Level-1 Trigger system after the phase-I upgrade [1]. The Global Trigger is the final step of the CMS Level-1 Trigger and implements a trigger menu, a set of selection requirements applied to the final list of objects from calorimeter and muon triggers [2]. The conditions for trigger object selection including topological requirements on multi-object triggers, invariant-mass calculations and other complex operations are combined by simple combinatorial logic to form the algorithms. The most basic algorithm consists of applying $E_{T}$ or $p_{T}$ threshold to a single object.

The LHC has resumed its operation in 2015 with the collision-energy of $13 \mathrm{TeV}$. The luminosity is expected to go up to $2 \times 10^{34} \mathrm{~cm}^{-2} \mathrm{~s}^{-1}$ during the period of LHC Run 2. The CMS Level-1 trigger system will be upgraded to improve its performance for selecting interesting physics events and to operate within the predefined data-acquisition rate in the challenging environment at LHC Run 2.

The Global Trigger will be re-implemented on modern FPGAs on an Advanced Mezzanine Card in a MicroTCA crate. The upgraded system will benefit from the ability to process complex algorithms with DSP slices and increased processing resources with optical links running at $10 \mathrm{Gbit} / \mathrm{s}$, enabling more algorithms at a time than previously possible and allowing CMS to be more flexible in how it handles the trigger bandwidth.

In order to handle the increased complexity of the trigger menu to be implemented on the upgraded Global Trigger as well as to make the menu implementation more flexible, a set of rules has been defined to express Level-1 algorithms. The system to realise the trigger menu on FPGA from Level-1 trigger algorithms expression based on the rules has been developed [3]. The design and implementation of the system for preparing a menu for the upgraded CMS Global Trigger will be presented.

\section{Level-1 menu grammar}

In order to make future evolutions of the Global Trigger possible, a set of rules for expressing algorithms on the Global Trigger (Level-1 menu grammar) has been introduced. By using the Level-1 menu grammar, the underlying hardware implementation is hidden. Also a new type of operation on trigger objects as well as a new type of trigger object itself can be used in an algorithm by updating the grammar without changing data structure to store the Level-1 menu information.

An algorithm is comprised of a set of object, cut, function, external signal and logical operators. An object is a physical object produced by the calorimeter and the muon trigger systems, i.e $\mu, e \gamma$, jet, $\tau, E_{T}, H_{T}, E_{T \text { miss }}$ and $H_{T \text { miss. }}$. In the Level-1 menu grammar, each object has its $p_{T}$ or $E_{T}$ threshold applied at a certain bunch crossing offset with respect to the bunch crossing ID of a collision. A function is a logical or mathematical computation based on objects. A cut is a condition applied on objects or on the value computed by a function. An external signal is a pre-defined set of binary input signals from sub-detectors to the Global Trigger. Figure 2 shows 
Calorimeter Trigger

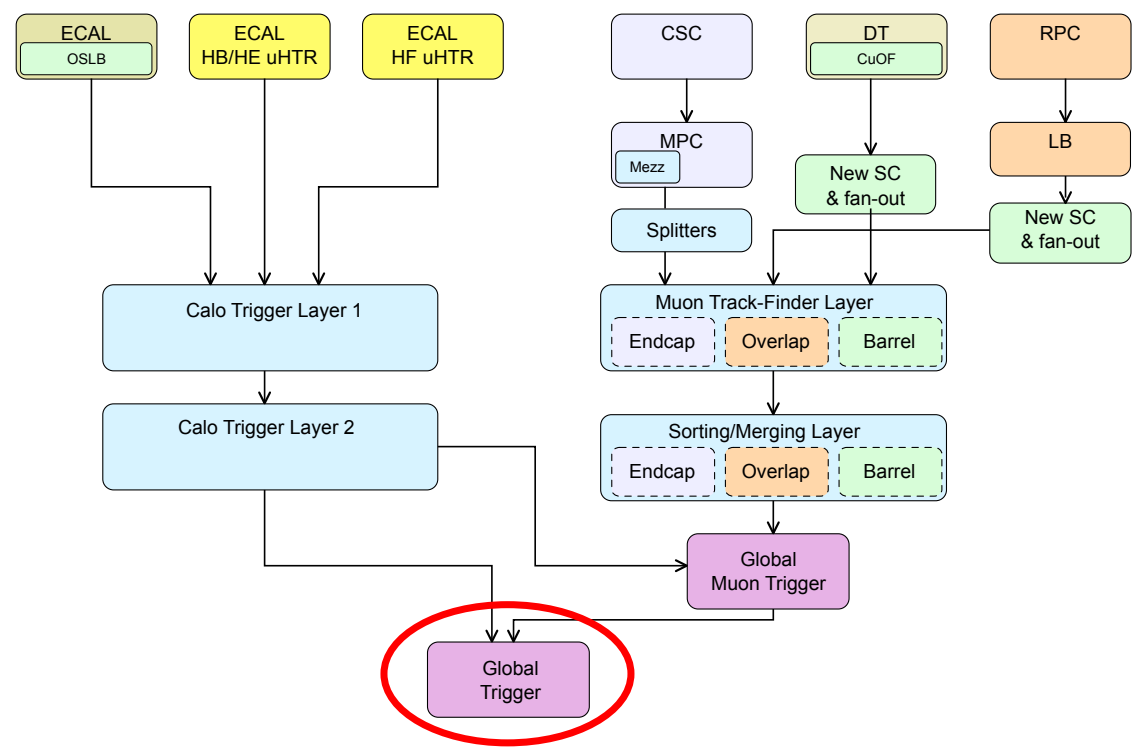

Figure 1: Block diagram of the CMS Level-1 trigger system after the phase-I upgrade

the algorithm expression for an arbitrary jet trigger with a $E_{T}$ threshold of $105.5 \mathrm{GeV}$ at a bunch crossing offset of +2 with $\eta$-range restriction. More details of the Level-1 menu grammar can be found elsewhere [3].

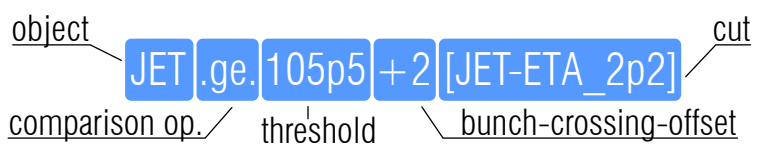

Figure 2: An example of algorithm expression.

\section{Level-1 trigger menu tools}

Software tools have been developed for implementing a grammar based Level-1 trigger menu on the Global Trigger. Figure 3 shows the tools for the trigger menu implementation. The three circles in the figure represent three software domains, i.e. online, offline and firmware domains. The Menu GUI provides a graphical user interface for a physicist to edit or to create a Level-1 trigger menu without exposing the complex hardware logic of the Global Trigger. The resulting Level-1 menu is then stored in the Menu XML file as a transient storage. The menu is translated to VHDL codes with VHDL producer in the firmware domain. The resulting codes will be synthesised to build a firmware which will then be deployed for online event selection. The contents of Menu XML will be stored in the online database for persistency using DB GUI in the online domain. The menu stored in the online database is used for data taking purpose and the menu is transferred to the offline database with the $\mathrm{O} 2 \mathrm{O}$ process in the boundary of the online and the offline domains. In the offline domain, the menu stored in the offline database is used for the Level-1 trigger emulator 
in CMS offline software framework. The emulator will use the Menu XML while a Level-1 trigger menu is being developed.

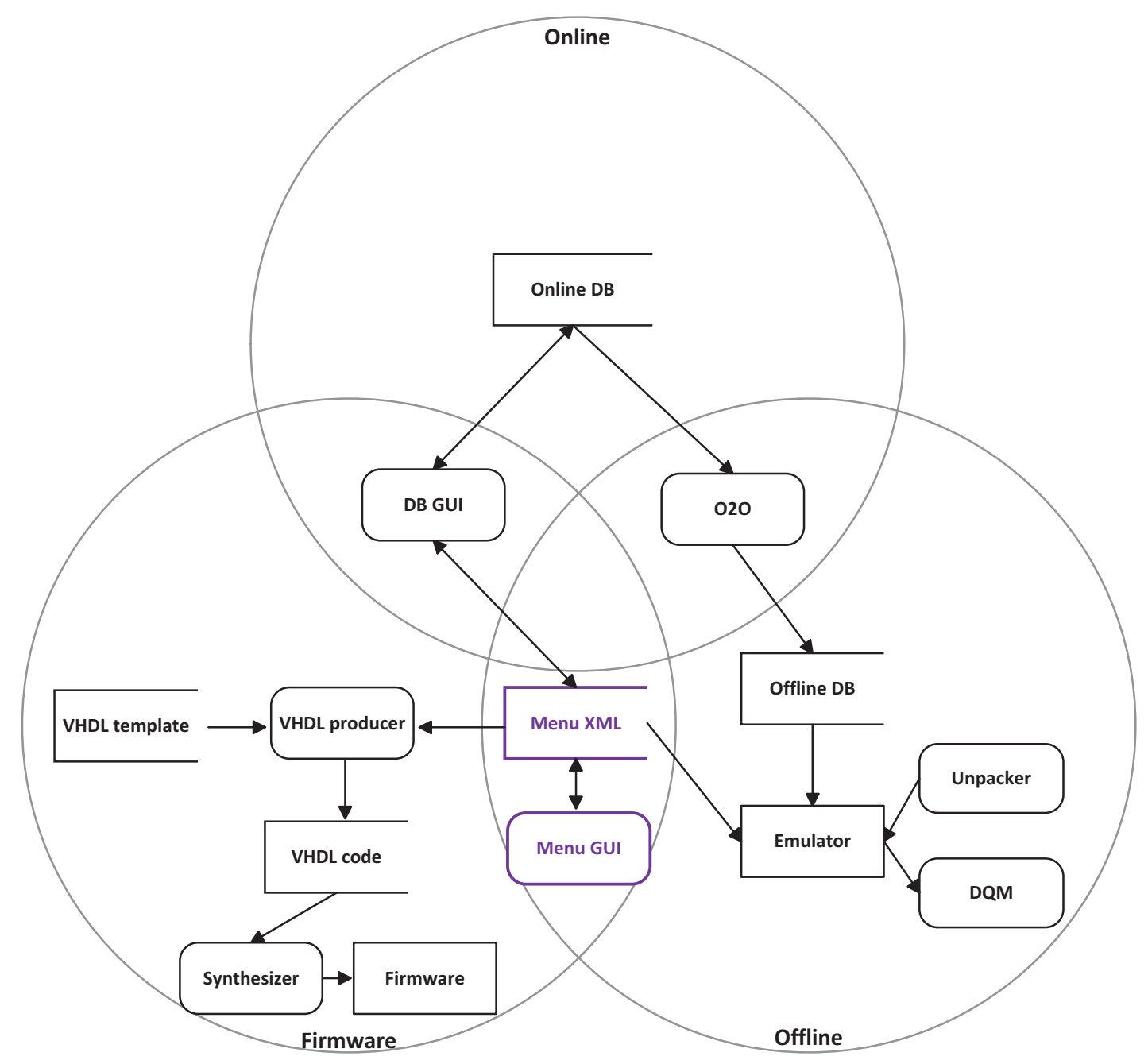

Figure 3: Dataflow diagram of Level-1 trigger menu tools based on the Gane-Sarson notation.

\section{Summary}

The Level-1 Trigger system of the CMS experiment will be upgraded for LHC Run 2, with emphasis on standardising hardware components and enhancing flexibility. The Global Trigger will be re-implemented on modern FPGAs on an Advanced Mezzanine Card in a MicroTCA crate. In order to make a Level-1 trigger algorithm implementation on the Global Trigger flexible, a set of rules for describing Level-1 trigger algorithms has been developed. Software tools have been implemented to help realising the event selection algorithms of increased complexity expressed with the set of rules on the Global Trigger. 


\section{Acknowledgements}

We acknowledge the enduring support for the construction and operation of the LHC and the CMS detector provided by the Bundesministerium für Wissenschaft, Forschung und Wirtschaft (Austria).

\section{References}

[1] CMS Collaboration 2012 CMS technical design report for the level-1 trigger upgrade" CMS-TDR-012

[2] M Jeitler et al. 2007 JINST 2 P01006

[3] T Matsushita et al. Software for implementing trigger algorithms on the upgraded CMS Global Trigger System, CMS CR-2015/083 to appear in IOP Conference Series 